\title{
Focusing and transport of high-intensity multi-MeV proton bunches from a compact laser-driven source
}

\author{
S. Busold, ${ }^{1, *}$ D. Schumacher, ${ }^{2, \dagger}$ O. Deppert, ${ }^{1}$ C. Brabetz,${ }^{3}$ S. Frydrych, ${ }^{1}$ F. Kroll,,${ }^{4,5}$ M. Joost, ${ }^{4}$ H. Al-Omari, ${ }^{3}$ \\ A. Blažević, ${ }^{2,6}$ B. Zielbauer, ${ }^{2,6}$ I. Hofmann, ${ }^{2,6}$ V. Bagnoud, ${ }^{2,6}$ T. E. Cowan, ${ }^{4,5}$ and M. Roth ${ }^{1}$ \\ ${ }^{1}$ Institut für Kernphysik, Technische Universität Darmstadt, Schloßgartenstraße 9, D-64289 Darmstadt, Germany \\ ${ }^{2}$ GSI Helmholtzzentrum für Schwerionenforschung, Planckstraße 1, D-64291 Darmstadt, Germany \\ ${ }^{3}$ Institut für angewandte Physik, Johann-Wolfgang-Goethe Universität Frankfurt, \\ Max von Laue Straße 1, D-60438 Frankfurt, Germany \\ ${ }^{4}$ Helmholtz-Zentrum Dresden-Rossendorf, Bautzner Landstraße 400, D-01328 Dresden, Germany \\ ${ }^{5}$ Institut für Kern- und Teilchenphysik, Technische Universität Dresden, Zellescher Weg 19, D-01069 Dresden, Germany \\ ${ }^{6}$ Helmholtz Institut Jena, Helmholtzweg 4, D-07734 Jena, Germany
}

(Received 18 April 2013; published 17 October 2013)

\begin{abstract}
Laser ion acceleration provides for compact, high-intensity ion sources in the multi-MeV range. Using a pulsed high-field solenoid, for the first time high-intensity laser-accelerated proton bunches could be selected from the continuous exponential spectrum and delivered to large distances, containing more than $10^{9}$ particles in a narrow energy interval around a central energy of $9.4 \mathrm{MeV}$ and showing $\leq 30 \mathrm{mrad}$ envelope divergence. The bunches of only a few nanoseconds bunch duration were characterized $2.2 \mathrm{~m}$ behind the laser-plasma source with respect to arrival time, energy width, and intensity as well as spatial and temporal bunch profile.
\end{abstract}

DOI: $10.1103 /$ PhysRevSTAB.16.101302

PACS numbers: 52.38.Kd, 41.75.Jv, 52.59.-f

\section{INTRODUCTION}

During the past decade, laser ion acceleration became a reliable tool for the production of intense ion beams in the multi-MeV range. The most investigated and robust acceleration mechanism is the target normal sheath acceleration (TNSA, [1,2]), typically used for accelerating protons. It delivers high-intensity bunches with ultralow emittances [3] and currently achievable proton energies of up to $70 \mathrm{MeV}$. Over the past years, numerous experimental investigations on the different facets of TNSA as well as simulation studies and theoretical approaches $[4,5]$ contributed to the deeper understanding of the mechanism and increasingly raised interest in the field. There is a wide and growing range of applications for these beams, e.g., already today the creation of warm dense matter [6-8], proton imaging [9], or the creation of bright neutron sources $[10,11]$, and possibly in the future proton fast ignition [12], clinical applications [13,14], or highintensity ion sources in general, in some cases combined with a postacceleration stage $[15,16]$. Special interest is given to the application as a diagnostic tool for high density matter, which is foreseen for experiments of the HEDgeHOB collaboration at the FAIR facility [17].

\footnotetext{
*Corresponding author.

s.busold@gsi.de

$\dagger$ de.schumacher@gsi.de

Published by the American Physical Society under the terms of the Creative Commons Attribution 3.0 License. Further distribution of this work must maintain attribution to the author(s) and the published article's title, journal citation, and DOI.
}

However, there are drawbacks with respect to the TNSA mechanism, namely (a) the exponential shape of the energy spectrum with the full energy spread (continuous spectrum up to a cutoff energy) and (b) the large initial divergence of the beam. Most of the possible future applications require the selection of a specific particle energy with $\Delta E / E<1 \%$ and a focused or low-divergent beam. Here, several attempts have been taken in the past and the most promising results obtained with the use of small permanent-magnetic quadrupole devices $[18,19]$ or pulsed high-field solenoids $[20,21]$.

The prospect of delivering a laser-accelerated proton beam, matched for further applications like injection into conventional acceleration infrastructure, initialized the formation of the LIGHT collaboration, i.e., the joint investigation of laser ion generation, handling, and transport. The collaborating institutes are, namely, TU Darmstadt, GSI, HZDR, JWGU Frankfurt, and HIJ (see list of affiliations above) and provide the necessary expertise in laser, plasma, and accelerator physics as well as pulsed magnet technology. The central goal is to build a demonstration beam line at GSI Darmstadt, where an ion optical focusing device (pulsed high-field solenoid or a doublet/triplet, compare [22]) is used to capture and energy select laser-accelerated protons in a first stage, and then perform a phase rotation with a radio-frequency (rf) cavity to either minimize the energy spread of the bunch or perform longitudinal focusing to reach highest intensities. Bunch intensities of up to $10^{10}$ particles at a central energy of $10 \mathrm{MeV}$ in less than a nanosecond are aimed for. 
The collaboration covers simulations as well as experiments and intensively works on further optimization of the TNSA process, in particular, as well as the exploration of other acceleration mechanisms in general. However, this paper will focus on the status of the experimental test stand at GSI and the latest experimental results.

\section{SETUP}

The experiments were performed with the PHELIX laser at GSI Darmstadt [23] at the Z6 experimental area. A dedicated beam line was constructed, providing up to $50 \mathrm{~J}$ of laser energy in $650 \mathrm{fs}$. The laser was focused to a $3.5 \times 3.5 \mu \mathrm{m}^{2}$ (FWHM) spot on target (flat gold foils with 5-10 $\mu \mathrm{m}$ thickness) and exceeded intensities of $10^{19} \mathrm{~W} / \mathrm{cm}^{2}$. Proton acceleration via the TNSA mechanism up to maximum energies of $28.4 \mathrm{MeV}$ could be demonstrated during the campaign.

The laser-accelerated proton beam entered a pulsed highfield solenoid, located $8 \mathrm{~cm}$ behind the target. The solenoid was $15 \mathrm{~cm}$ long with a clear aperture of $40.5 \mathrm{~mm}$. It was connected to a pulse power supply unit, which could drive up to $10.4 \mathrm{kA}$ through the solenoid, resulting in a maximum magnetic field of $8.7 \mathrm{~T}$. The time-depending magnetic field evolution can be described by an ideal LC circuit with a time constant $\tau=\sqrt{\mathrm{LC}}=0.2 \mathrm{~ms}$. Therefore, the magnetic field can be assumed constant for the passing protons (ns time scale). The electrical circuit was critically damped with a crowbar diode array to prevent oscillations. A great advantage of the pulsed device is the possibility of flexible adjustment of the field strength.

Two main diagnostics were used to characterize the proton beam: radiochromic films (RCF) in stacked configuration and a diamond detector for time-of-flight measurements.

The RCFs have a thin proton-sensitive layer, which changes its color "blueish" due to the deposited energy of the protons. Characterization of laser-accelerated proton beams with RCFs in stacked configuration is a well established technique (RIS, [24]) yielding important parameters like the shape of the energy spectrum, the absolute proton numbers, and the energy dependent envelope divergence of the beam. It also gives a high resolution transverse beam profile for several energies within the spectrum of the beam, as different energies are stopped in different layers of the RCF stack and have their highest differential energy loss in their stopping layers (Bragg peak behavior).

A diamond detector as a complementary diagnostic measured the temporal beam profile in time-of-flight configuration. It consisted of four diamond segments of $4 \times 4 \mathrm{~mm}^{2}$ area with a thickness of $20 \mu \mathrm{m}$. They were arranged to a $8 \times 8 \mathrm{~mm}^{2}$ detection array. Protons passing through this array excite electron-hole pairs and the charge carriers are drained due to an applied voltage of $10 \mathrm{~V}$, resulting in a detectable signal. The time-of-flight technique allows for translating the proton arrival time into energies, as the whole beam can be considered produced "instantly" at

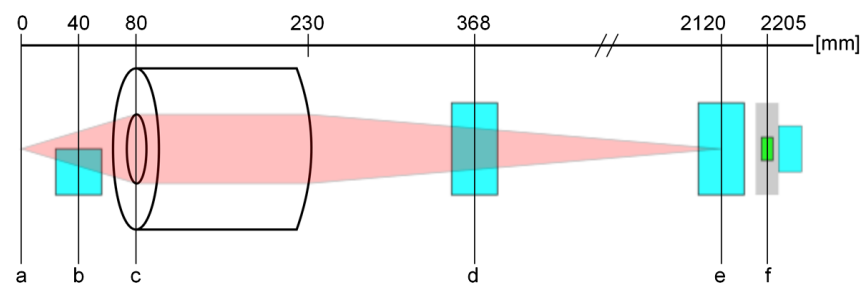

FIG. 1. Experimental setup. After the protons are produced in the laser-matter interaction at the target (a), the divergent bunch enters the solenoid (c). For characterization of the initial beam, a RCF stack can optionally be placed in front of the solenoid at $40 \mathrm{~mm}$ (b). For detection of the bunch at larger distances, either a RCF stack can be used for dose analysis and transverse beam profile measurements at distances up to $2120 \mathrm{~mm}$ (d),(e) or alternatively a diamond detector at $2205 \mathrm{~mm}$ for time-of-flight measurements with also a RCF stack attached to detect the protons after passing the $20 \mu \mathrm{m}$ thin diamond (f).

the time the laser pulse hits the target (acceleration time is in the order of the laser pulse length, here less than $1 \mathrm{ps}$ ). The voltage was applied to the diamond through bias tees and the signal was damped by $5 \mathrm{~dB}$. Figure 1 shows the experimental setup.

Detection of the beam was possible at different distances up to $2.2 \mathrm{~m}$ from the source. The characterization of the initially produced proton spectrum can practically be done only in close distance to the source, as the beam is highly divergent. Therefore, one RCF stack was placed $4 \mathrm{~cm}$ behind the target. As the beam is absorbed by the stack, only a half-size stack was used for detecting half of the initial beam while the other half entered the solenoid and could be analyzed at a later position by another RCF stack, respectively, the diamond detector.

During the campaign energy selection and beam transport to distances larger than $2 \mathrm{~m}$ were performed. The main objective was the bunch characterization in arrival time, energy width, divergence, and intensity at this position far from the source, thus demonstrating the creation of a laseraccelerated proton bunch that is ready for injection into the rf cavity and delivering highest beam intensities.

The experiments were accompanied by simulation studies using the TRACEWIN code [25]. Because of the chromaticity of the solenoid there are high particle losses to the wall of the beam line and inside the solenoid. Hereby, an efficient energy filtering is achieved intrinsically and the initial exponential spectral shape of the beam is transformed into a peaked shape around the focused energy. This process is well described in e.g. [14]. In our case, a realistic initial TNSA proton distribution (as produced in the here presented experimental campaign) was used to simulate the transport process and the expected distribution behind the solenoid at larger distances from source for comparison to the experimental data. The expected transverse beam profile and the $x-x^{\prime}$ phase space at $2.2 \mathrm{~m}$ from source are shown in Fig. 2. The simulation used a custom field distribution for the solenoid magnetic field, which fits the measured real field distribution. 

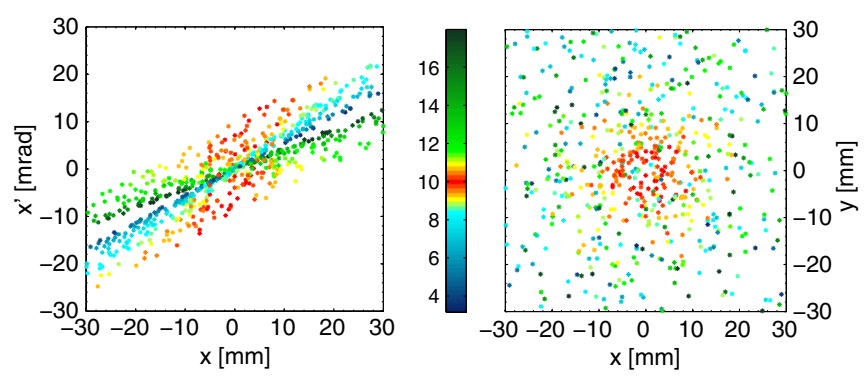

FIG. 2. Simulated phase space and transverse beam profile of a laser-accelerated proton beam at a distance of $2.2 \mathrm{~m}$ behind the source. The particles are color coded due to the adjustment of the solenoid to focus protons of $10 \mathrm{MeV}$ at this position.

\section{RESULTS}

For comparison of the experimental results with simulations and investigation of the transport efficiency, the solenoid was set to $\mathrm{B}_{\max }=7.35 \mathrm{~T}$, which focused protons of $9.4 \mathrm{MeV}$ to a position at $2.12 \mathrm{~m}$ behind the laser-plasma source. At this distance only a small fraction of the spectrum was still detectable. Lower energies were focused at smaller distances and diverge again afterwards, while the solenoid field was not strong enough to focus higher energies, which were consequently still diverging. For a detailed investigation of the transport properties of the solenoid, closer distances were more favorable, as here a wide range of energies was still detectable. Therefore, the transported beam was detected with a RCF stack first at a position of $36.8 \mathrm{~cm}$ behind the source, while at the same time another (half-aperture-sized) RCF stack measured the initial beam at $4 \mathrm{~cm}$ distance as reference. Figure 3 shows the proton imprint of the half beam at $36.8 \mathrm{~cm}$ in the different layers of the RCF stack. These can be compared with the results of the simulations and show a good agreement with respect to beam size and rotation angle of better than $10 \%$. Focusing power and rotation (counterclockwise direction for Fig. 3) are stronger towards lower proton energies; the lowest energies already passed their focal spot and are divergent again (resulting in a vertically mirrored imprint).

Concerning the transport efficiency, the maximum capture angle of the solenoid at this position has to be taken

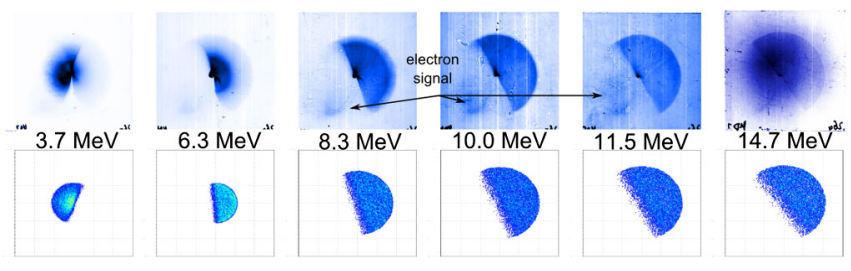

FIG. 3. Proton imprint in the RCF stack at $36.8 \mathrm{~cm}$ behind the source: experimental (upper) and simulation (lower sequence) results. In both cases the size of a single film is $60 \mathrm{~mm} \times$ $60 \mathrm{~mm}$. Additionally, an electron signal can be seen in the experimental data in the lower left quarter. into account. While the initial beam shows a half opening angle of $350 \mathrm{mrad}$ for $10 \mathrm{MeV}$ protons, the solenoid is only able to capture particles with less than $100 \mathrm{mrad}$ half opening angle. However, the Gaussian-like transverse beam profile results in a higher particle density in the center thus improving the situation. Our measurements show up to $34 \%$ transmitted protons, which is quantitatively $1.1 \times 10^{9}$ out of the initial $3.2 \times 10^{9}$ protons in the energy interval of $(9.8 \pm 0.5) \mathrm{MeV}$ for the shot shown in Fig. 3.

At larger distances, a characterization of the bunch was done with the full beam. Typical shot-to-shot fluctuations of up to a factor of 3 in particle numbers were measured during the campaign and have to be taken into account. Figure 4 shows the transverse focus profile when (a) focusing $9.4 \mathrm{MeV}$ or (b) $5 \mathrm{MeV}$ protons at $1.07 \mathrm{~m}$ distance to source, using field strengths of 7.79 and $5.23 \mathrm{~T}$, respectively. The focal spot contained (a) $4 \times 10^{8}$ and (b) $1.6 \times 10^{9}$ protons and was about $5 \times 5 \mathrm{~mm}^{2}$.

Figure 5(a) shows the proton focus at a distance of $2.12 \mathrm{~m}$ to the source in the RCF layer, which corresponds
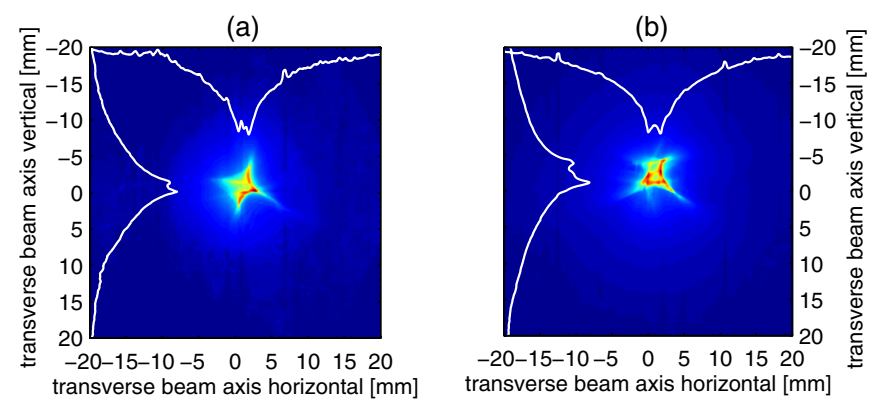

FIG. 4. Measured transverse beam profile of the focused energy (a) $9.4 \mathrm{MeV}$ and (b) $5 \mathrm{MeV}$ at a distance to the source of $1.07 \mathrm{~m}$. In both cases the spot was around $5 \mathrm{~mm}$ in diameter and contained (a) $4 \times 10^{8}$ and (b) $1.6 \times 10^{9}$ protons.
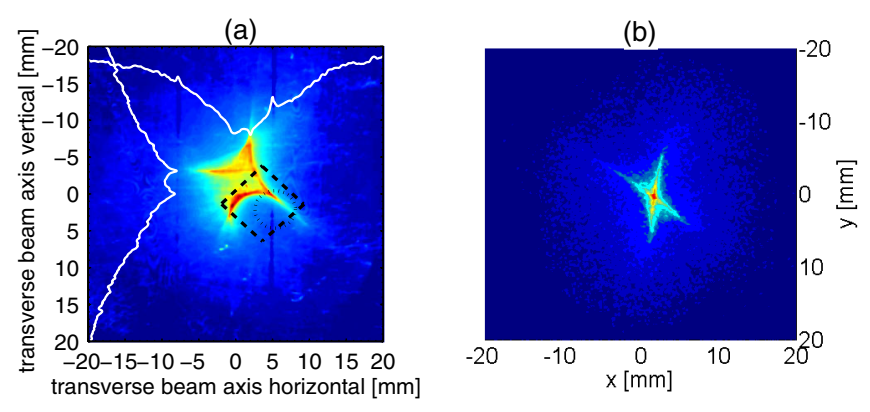

FIG. 5. (a) Measured transverse focus profile of $9.4 \mathrm{MeV}$ protons behind the solenoid at $2.12 \mathrm{~m}$ distance to source. The focus is about $10 \mathrm{~mm}$ in diameter and contains $5 \times 10^{8}$ protons. (b) Simulated focal spot in the corresponding RCF layer, using a realistic wire assembly for the solenoid coil, which results in an observable astigmatism of the lens. Additionally indicated are the position of the diamond detector (dashed black square) and the optional $5 \mathrm{~mm}$ aperture (dashed black circle) relative to the proton beam. 
to a Bragg penetration depth of $9.4 \mathrm{MeV}$ protons. The focal spot size increased compared to the $1.07 \mathrm{~m}$ position due to the weaker focusing to about $1 \mathrm{~cm}^{2}$ in size and contains $6.5 \times 10^{8}$ protons in this case. The squarelike shape of the focus is due to field aberrations of the solenoid; compare also [21]. Modeling the exact arrangement of the coil wire leads to a lens aberration (astigmatism) of the solenoid and to the observed shape. This is shown in Fig. 5(b). Contrary to the expectations from the simulations, the RCF layers corresponding to higher energies $(>10 \mathrm{MeV})$ showed also an observable focal feature up to the last film with detectable signal. Figure 6 displays the RCF imprints, while in Fig. 7 the quantitative results are shown. From the experimental data a proton spectrum of the shape $d N / d E=N_{0} / E \times$ $\exp (-E / k T)$ was deduced with the parameters $N_{0}=$ $(1.21 \pm 0.21) \times 10^{11}$ and $k T=(2.55 \pm 0.09) \mathrm{MeV}$. The initial proton spectra, fitted to the same function, show a higher value for $k T$ in the order of $6.5-7.0 \mathrm{MeV}$, which indicates that only a subpopulation of the higher energetic protons are contributing to this focal feature. Before discussing these findings in the next section, the results of the diamond detector as a complementary diagnostic will be presented as they can confirm the measurements.

The diamond detector at $2.2 \mathrm{~m}$ distance to the source was used for a temporal characterization of the bunch. Via time-of-flight (ToF) the recorded electrical signal can be translated into a proton spectrum. The energy spectra were clearly peaked around the focused energy for each shot, see Fig. 8 (one should be aware that the signal chronologically starts from right with the higher energetic protons first arriving and then evolves to the left). Transforming from time to energy, however, shows an artificial increase
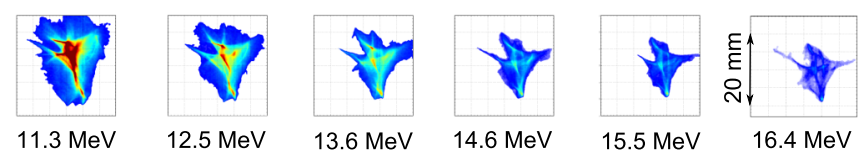

FIG. 6. Proton imprint (in units of deposited energy) in the $\mathrm{RCF}$ layers for energies larger than the focused one with corresponding Bragg energies. Contrary to the expectations, a focused feature is still detectable up to the last layer.

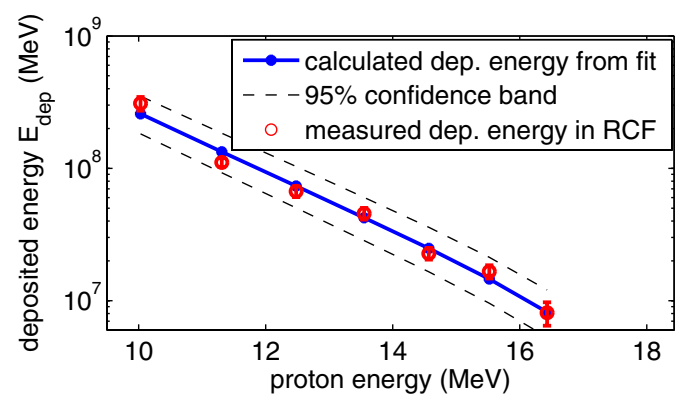

FIG. 7. Measured deposited energy (red circles) in RCFs when focusing $10 \mathrm{MeV}$ protons at $2.12 \mathrm{~m}$ and corresponding exponential fit (blue line).

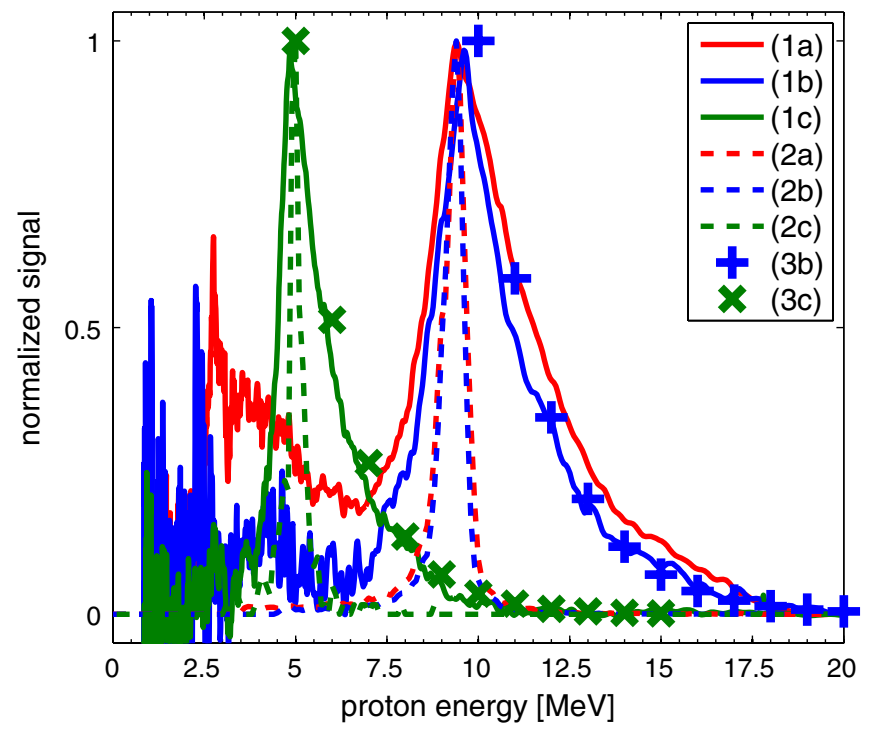

FIG. 8. Normalized time-of-flight results of the diamond detector (at $2.2 \mathrm{~m}$ from source) for three different shots: (1a) focusing of $9.4 \mathrm{MeV}$ protons, (1b) focusing of $9.4 \mathrm{MeV}$ protons with a $5 \mathrm{~mm}$ pinhole at $2.12 \mathrm{~m}$, and (1c) focusing of $5 \mathrm{MeV}$, also using the pinhole. The dashed graphs (2a)-(2c) are the corresponding expected spectra from simulations. (3b) and (3c) show the exponential decaying spectrum for the high energies obtained from the RCF data.

towards lowest energies due to the conversion $\Delta t \rightarrow \Delta E$. The sudden drop at $2.5 \mathrm{MeV}$ was due to a $20 \mu \mathrm{m}$ thin copper foil in front of the diamond; it was necessary for shielding against the x-ray background, but also blocked lower energetic protons. Shown in Fig. 8 are the measured ToF signals [(1a)-(1c)], the simulated ones [(2a)-(2c)] and the reference spectra from the RCFs of the corresponding shots [(3b) and (3c)]. Because of detector size and experimental setup, the diamond detector measured only a subaperture of the beam. This fraction could be further narrowed by putting a $5 \mathrm{~mm}$ diameter aperture in front. Exact positions of diamond and aperture with respect to the beam are indicated in Fig. 5 .

While hitting the focused energy with the intensity peak very good [9.4 MeV for graphs (1a) and (1b), and $5 \mathrm{MeV}$ for (1c)], one can on one hand observe a relative reduction of lower energetic protons when applying the aperture and on the other hand a disagreement in energy width between simulated and measured spectra. In bunch lengths (FWHM), this corresponds to rather large values of $8.2 \mathrm{~ns}$ (1a) and $6.5 \mathrm{~ns}(1 \mathrm{~b})$ compared to $2.1 \mathrm{~ns}$ [(2a),(2b)] for 9.4 MeVand $11.2 \mathrm{~ns}(1 \mathrm{c})$ compared to $1.7 \mathrm{~ns}(2 \mathrm{c})$ for $5 \mathrm{MeV}$.

With the protons passing the only $20 \mu \mathrm{m}$ thin diamond nearly undisturbed, they could be measured with the RCF technique simultaneously, placing a RCF stack directly behind the diamond. An exponential decay from peak energy to higher energies, deduced from the RCFs, is also plotted in Fig. 8, graphs (3b) and (3c), and fits the diamond signal quite well. 


\section{DISCUSSION}

A discrepancy between our simulations and the obtained experimental data was found: the energy widths of the proton bunches were much broader than in the calculations and a focuslike feature was visible throughout the higher energies. This longitudinal broadening was consistently measured with the RCFs and the diamond detector towards the high energies, while it was observed towards lower energies in the diamond signal only, as in the RCFs this effect was hardly visible due to the also present imprint from the higher energetic protons within the first films.

An error of the focusing field can be excluded. The field distribution had been measured (with DC current) and found to be in good agreement with simulations. From dynamic effects only a slight decrease in maximum field strength and a temporal shift between current and magnetic field is to be expected due to the occurrence of eddy currents (in the order of $0.04 \mathrm{~ms}$ ). Finally, the correct focusing of the proton energies is the strongest indicator for a mainly undisturbed field.

A possible explanation can be found by taking into account the effect of the copropagating electrons. Inside the solenoid field, they are tightly focused on axis and thus build up a negative potential. Earlier simulations already predicted this effect [26]. For a first estimate for the experiment at hand, some considerations are done: The gyroradius of copropagating electrons (energies of a few $\mathrm{keV}$ ) in the solenoid is of the order of $50 \mu \mathrm{m}$. Therefore the electrons will be contained in a cylinder of this radius inside the solenoid. First simulations with a static potential on the solenoid center (against the inner wall of the solenoid) start showing an effect on the protons at values for the potential of $>5 \mathrm{kV}$. Choosing the model of a cylinder condensator for the electron cylinder inside the stainless steel beam tube, which covers the inner side of the solenoid, one can estimate the number of electrons $N$ needed to create such a potential: $N=\frac{C \times U}{e}=\frac{2 \pi \epsilon_{0} \times l \times U}{e \times \ln (R / r)}$ with $e=$ $1.6 \times 10^{-19} \mathrm{Cb}$ the electron charge, $U=5 \mathrm{kV}$ the potential, and $C=2 \pi \epsilon_{0} \frac{l}{\ln (R / r)}$ the capacity $\left(\epsilon_{0}=\right.$ $8.85 \times 10^{-12} \mathrm{As} / \mathrm{Vm}$ the electrical field constant, $l=0.15 \mathrm{~m}$ the length of the solenoid, $r=50 \mu \mathrm{m}$ and $R=2 \mathrm{~cm}$ the small and large radii). This simple estimate gives $N=4 \times 10^{10}$ electrons, which is quite in the right order of magnitude for the minimum necessary electron number. Such an electrical field inside the solenoid may be able to provide the necessary focusing force to explain the experimental findings of a focuslike feature in the energies higher than the focused energy.

Additionally, hints to the existence of this electron induced electric field inside the solenoid can be found in two other features of the experimental data. First in the transverse beam profile of the focal energy (see Fig. 5): in the experiment, protons seem to be deflected from the exact center. A simple static simulation approach as described above results in such a behavior, as inside the solenoid protons near axis see a higher additional focusing field due to the electrons than protons on larger radii. This leads to an earlier focus of the near-axis protons compared to protons of the same energy but on greater radii. Overlapping both those proton populations results in a depleted central region, which was observed in the RCF.

A second hint for an additional field inside the solenoid is observable in Fig. 3: There is a small but intense spot within the beam on the center axis throughout all detected energies. While an imprint of high energetic electrons is also visible in the films, this feature belongs to the proton beam and cannot be explained by the solenoid magnetic field.

\section{SUMMARY AND OUTLOOK}

Energy selection and transport of intense laseraccelerated proton bunches to distances up to $2.2 \mathrm{~m}$ was demonstrated. Because of the flexibility of the pulsed highfield solenoid, the central energy of a bunch could easily be selected from the initial spectrum; here shown were lowdivergent bunches of 5 and $9.4 \mathrm{MeV}$ central energy in a narrow peaked spectrum, containing proton numbers above $10^{9}$ particles in a few ns bunch length. The transverse beam profile was measured and the temporal bunch characterization obtained as well as the precise arrival time of the bunch.

Furthermore, an achromatic focusing effect inside the solenoid was observed, which led to a broadening of the peaked proton spectrum. The copropagating electrons were identified as a possible reason for this effect. However, confirmation of this hypothesis by detailed simulation studies has not been done yet and needs to follow.

In the next step of the LIGHT project, a radio-frequency cavity will be installed at a distance of $2 \mathrm{~m}$ behind the source. Inside, the bunch will pass the potential of a 108.4 MHz electromagnetic field, which allows for two different scenarios depending on the strength of the $\mathrm{rf}$ wave: (a) reducing the energy spread of the bunch to prevent further longitudinal defocusing or (b) perform a phase focusing, resulting in the production of highest intensity bunches of sub-ns bunch lengths.

\section{ACKNOWLEDGMENTS}

The authors would like to thank the PHELIX laser team for their commitment to the LIGHT project and the highfield lab of HZDR for their work on the pulsed power equipment. This work is supported by HiC4FAIR.

[1] S. C. Wilks, A. B. Langdon, T. E. Cowan, M. Roth, M. Singh, S. Hatchett, M. H. Key, D. Pennington, A. MacKinnon, and R. A. Snavely, Phys. Plasmas 8, 542 (2001).

[2] R. A. Snavely, M. H. Key, S. P. Hatchett, T. E. Cowan, M. Roth, T.W. Phillips, M.A. Stoyer, E. A. Henry, T. C. 
Sangster, M.S. Singh, S.C. Wilks, A. MacKinnon, A. Offenberger, D.M. Pennington, K. Yasuike, A.B. Langdon, B. F. Lasinski, J. Johnson, M. D. Perry, and E. M. Campbell, Phys. Rev. Lett. 85, 2945 (2000).

[3] T. E. Cowan et al., Phys. Rev. Lett. 92, 204801 (2004).

[4] P. Mora, Phys. Rev. Lett. 90, 185002 (2003).

[5] M. Passoni and M. Lontano, Phys. Rev. Lett. 101, 115001 (2008).

[6] A. Pelka et al., Phys. Rev. Lett. 105, 265701 (2010).

[7] P. K. Patel, A. J. Mackinnon, M. H. Key, T. E. Cowan, M. E. Foord, M. Allen, D. F. Price, H. Ruhl, P. T. Springer, and R. Stephens, Phys. Rev. Lett. 91, 125004 (2003).

[8] G. M. Dyer, A. C. Bernstein, B. I. Cho, J. Osterholz, W. Grigsby, A. Dalton, R. Shepherd, Y. Ping, H. Chen, K. Widmann, and T. Ditmire, Phys. Rev. Lett. 101, 015002 (2008).

[9] A. J. Mackinnon et al., Rev. Sci. Instrum. 75, 3531 (2004).

[10] M. Roth et al., Phys. Rev. Lett. 110, 044802 (2013).

[11] S. Karsch, S. Düsterer, H. Schwoerer, F. Ewald, D. Habs, M. Hegelich, G. Pretzler, A. Pukhov, K. Witte, and R. Sauerbrey, Phys. Rev. Lett. 91, 015001 (2003).

[12] M. Roth, T.E. Cowan, M. H. Key, S.P. Hatchett, C. Brown, W. Fountain, J. Johnson, D. M. Pennington, R. A. Snavely, S. C. Wilks, K. Yasuike, H. Ruhl, P. Pegoraro, S. V. Bulanov, E. M. Campbell, M. D. Perry, and H. Powell, Phys. Rev. Lett. 86, 436 (2001).

[13] K. Nemoto, A. Maksimchuk, S. Banerjee, K. Flippo, G. Mourou, D. Umstadter, and V. Y. Bychenkov, Appl. Phys. Lett. 78, 595 (2001).

[14] I. Hofmann, J. M. ter Vehn, X. Yan, and H. Al-Omari, Nucl. Instrum. Methods Phys. Res., Sect. A 681, 44 (2012).
[15] A. Almomani, M. Droba, U. Ratzinger, and I. Hofmann, Phys. Rev. ST Accel. Beams 15, 051302 (2012).

[16] S. Sinigardi, G. Turchetti, P. Londrillo, F. Rossi, D. Giove, C. De Martinis, and M. Sumini, Phys. Rev. ST Accel. Beams 16, 031301 (2013).

[17] HEDgeHOB, http://hedgehob.physik.tu-darmstadt.de/ (2005).

[18] M. Schollmeier et al., Phys. Rev. Lett. 101, 055004 (2008).

[19] M. Nishiuchi et al., Appl. Phys. Lett. 94, 061107 (2009).

[20] K. Harres, I. Alber, A. Tauschwitz, V. Bagnoud, H. Daido, M. Gunther, F. Nürnberg, A. Otten, M. Schollmeier, J. Schutrumpf, M. Tampo, and M. Roth, Phys. Plasmas 17, 023107 (2010).

[21] T. Burris-Mog, K. Harres, F. Nürnberg, S. Busold, M. Bussmann, O. Deppert, G. Hoffmeister, M. Joost, M. Sobiella, A. Tauschwitz, B. Zielbauer, V. Bagnoud, T. Herrmannsdoerfer, M. Roth, and T.E. Cowan, Phys. Rev. ST Accel. Beams 14, 121301 (2011).

[22] I. Hofmann, Phys. Rev. ST Accel. Beams 16, 041302 (2013).

[23] V. Bagnoud et al., Appl. Phys. B 100, 137 (2010).

[24] F. Nürnberg, M. Schollmeier, E. Brambrink, A. Blazevic, D. C. Carroll, K. Flippo, D. C. Gautier, M. Geissel, K. Harres, B. M. Hegelich, O. Lundh, K. Markey, P. McKenna, D. Neely, J. Schreiber, and M. Roth, Rev. Sci. Instrum. 80, 033301 (2009).

[25] cea, http://irfu.cea.fr/sacm/logiciels/index3.php (2013).

[26] F. Nrnberg, A. Friedman, D. P. Grote, K. Harres, B. G. Logan, M. Schollmeier, and M. Roth, J. Phys. Conf. Ser. 244, 022052 (2010). 